\title{
Simulation on a partitioned urban network: an approach based on a network fundamental diagram
}

\author{
A. Briganti, G. Musolino \& A. Vitetta \\ DIIES - Dipartimento di ingegneria dell'Informazione, \\ delle Infrastrutture e dell'Energia Sostenibile, \\ Università degli Studi Mediterranea di Reggio Calabria, Italy
}

\begin{abstract}
The paper focuses on the estimation of multiple Network Fundamental Diagrams (NFDs) associated with clusters obtained after the partitioning of an urban transport network. At this stage, the network is partitioned according to the criterion of the spatial distribution of urban activities, in order to estimate NFDs for the centre and suburbs of the urban area. These operations are necessary to reduce the variance of link densities within each cluster, ensuring that the hypothesis of existence that a homogeneously shaped (with no hysteresis) NFD holds. The experimentation is developed in the town of Villa San Giovanni, in southern Italy. The preliminary results show that the shape of the NFDs seems to depend on the spatial distribution of travel demand.
\end{abstract}

Keywords: Network Fundamental Diagram, traffic flow theory, network partitioning, urban area.

\section{Introduction}

The existing methods for management and control (e.g. gating, pricing, signal setting) of congested urban networks generally operate with information about traffic conditions (e.g. vehicular flows, speeds, densities), which are disaggregated at link and junction levels. This implies, especially in dynamic contexts, the necessity to develop sophisticated architecture (see [1] for a description), composed of technological and simulation tools, in order to obtaining reliable and disaggregated estimations and forecasts of traffic conditions. 
An alternative line of research asserts that (aggregate) global traffic conditions of a network may be measured by an asymmetric inverse-U shaped diagram, called a Network Fundamental Diagram (NFD) by some authors. Other authors call it a Macroscopic Fundamental Diagram (MFD). The term 'network' in the definition is preferred to emphasize the distinction between the network-related diagram, as it is the NFD, and the link fundamental diagram, which are both macroscopic.

As reported in [2]: 'the basic idea (of NFD) consists of dividing the city into neighbourhood-size reservoirs (of dimensions comparable with a trip length) and to shift the modelling emphasis from microscopic predictions to macroscopic monitoring and control'. NFD puts in relation two aggregate variables: the first, called production, is defined as the product of average flow and network length, and the second, called accumulation, is defined as the product of density and network length. The seminal papers (see $[2,3]$ ) postulated the existence of NFD under the condition of (quasi)-homogeneously congested urban networks. This means that, if in (a cluster of) the network, the spatial distribution of link densities is similar in two different time intervals with the same average network density, these two time intervals should have the same average flows (in other words, these two points are close each other in a NFD plane). Geroliminis and Sun worked on the network of Yokoama (see [4]). They showed that their estimated NFD, related to the part of network monitored with detectors, is less-scattered than the ones of individual links. They also asserted that the NFD was estimated under different travel demand configurations.

Buisson and Ladier [5] investigated the impacts of heterogeneity of network characteristics on the existence of NFD, analysing data from loop detectors of the city of Toulouse (France). They concluded that elements of heterogeneity on the network, such as a different distance between detector and traffic signal, onset and offset congestion, roads belonging to central vs suburbs areas, urban vs highway roads, may generate hysteresis in the global NFD curve. Later, Ji and Geroliminis [6] introduced some algorithms to cluster urban networks, with the double objectives of reducing the variance of link densities within a cluster and to maintain the spatial compactness of each cluster in order to allow the application of perimeter control strategies.

Several papers used the concept of NFD for different purposes such as congestion control by means of gating $[7,8]$ and pricing schemes [9].

The objective of the paper is to estimate homogeneous well-shaped NFDs after the network partitioning, in order to reduce the variance of link densities within each cluster. The estimation of NFDs could allow the application of routing strategies [10] and, in general, support urban planning activities [11]. NFD diagrams are built by means of simulated data of the transport system of the town of Villa San Giovanni (Italy).

\section{Methodology}

The NFD requires the calculation of indicators on average (weighted) values of vehicular flow and density among links of the network (of a cluster of it).

The two indicators are defined as follows (see [3, 4]): 
- $\quad$ Average Flow (AF):

$$
A F=\Sigma_{j} f_{j} \cdot l_{j} / L \quad j \in A
$$

- $\quad$ Average Density $(A D)$ :

$$
A D=\Sigma_{j} k_{j} \cdot l_{j} / L \quad j \in A
$$

with

$\mathrm{f}_{\mathrm{j}}$, vehicular flow on link $j$;

$l_{j}$, link length on link $j$;

$k_{\mathrm{j}}$, density on $\operatorname{link} j$;

$L=\Sigma_{\mathrm{j}} l_{j}$, total network length;

$A$, set of links belonging to the network.

The above indicators may be estimated for the entire network or for a portion of it. In the second case, each link $\mathrm{j}$ belongs to a specific cluster: $j \in A$ ' with $A^{\prime} \subseteq A$.

The partitioning of the network into clusters arises from the necessity to have a more homogeneous NFD, with a reduction in the variance of density values for each cluster.

In our opinion, the shape of the NFD depends on the spatial distribution of travel demand. Therefore, a criterion for network partitioning, that could allow the demand flows to be distributed as "uniform as possible" among the links belonging to the cluster, could support the estimation of a well-shaped NFD. In this work, we have adopted an empirical criterion of network partitioning related to the level and the spatial distribution of activities inside the urban area, which is the generator of the spatial structure of the travel demand flows, which, in its turn, generate link flows and densities.

We have estimated the indicators for the whole network and for different clusters located in the central area and in the suburbs.

\section{Experimentation}

The indicators specified in section 2 are estimated for the urban transport system of Villa San Giovanni (Italy). The transport system is simulated by means of the microscopic traffic simulation package AIMSUN [12], which uses a within-day dynamic traffic assignment model (see [13] for details). The main elements of the transport system are described in subsection 3.1 and the preliminary results of the NFDs estimations in subsection 3.2.

\subsection{Transport system and network partitioning}

The network of the urban area of Villa San Giovanni has 358 links and 134 nodes and it has a total length of $63 \mathrm{~km}$.

The average value of capacity of each link is 1200 vehicles/hour. The study area is subdivided into 29 traffic zones and it is partitioned into 3 clusters, 
according to the criterion related to the level and the spatial distribution of activities. The three clusters are:

- $\quad$ cluster A, which identifies the southern outskirts of the town, with 52 road links and a total length of about $9 \mathrm{~km}$;

- $\quad$ cluster B, which identifies the central district of the town, with 193 road links and a total length of about $19 \mathrm{~km}$;

- cluster $\mathrm{C}$, which identifies the northern outskirts of the town, with 113 links and a total length of about $35 \mathrm{~km}$.

Figure 1 depicts the graph of the network and its partitioning into three clusters.

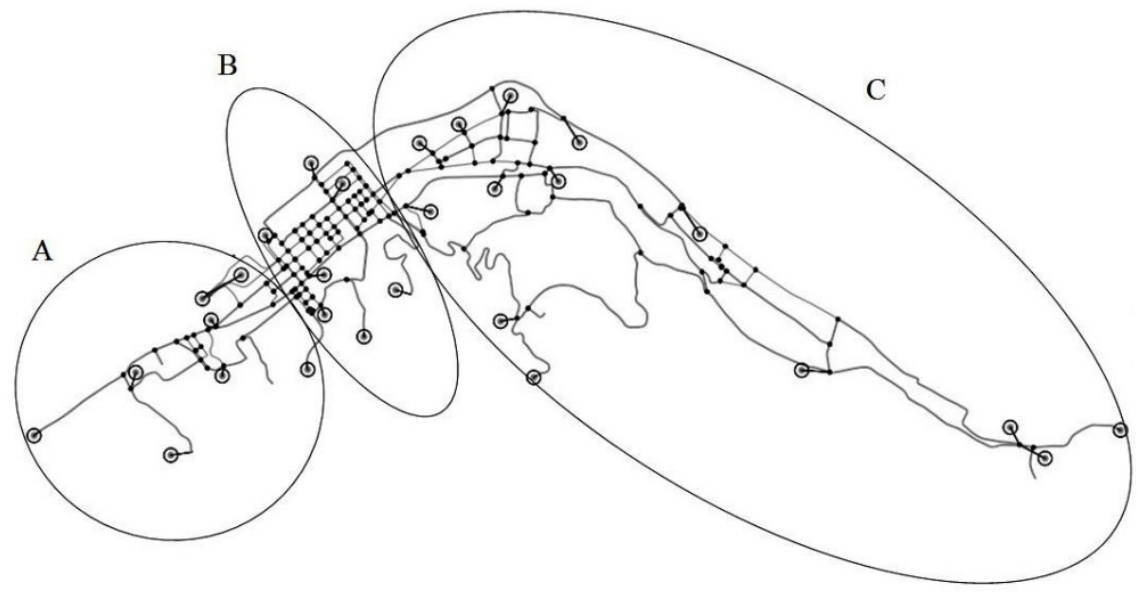

Figure 1: Network and partitioning of urban area of Villa San Giovanni (Italy).

The origin-destination travel demand matrix is estimated in this paper by means of a multi-stage model, with considers emission, distribution and modal choice [14].

The path choice model is a behavioral model, which estimates the probability that an individual user undertakes a trip along a path connecting an origin destination pair. The path choice is pre-trip and the probability is estimated on the basis of travel times on the network updated every 10 seconds. The value of the probability of choosing a path has been estimated by means of a C-logit path choice model [15].

\subsection{Estimation of NFDs}

The NFDs for the network of Villa San Giovanni are estimated by simulating the transport system for a period of 5 hours (subdivided into time slices of 5 minutes). Two scenarios for travel demand are assumed:

- uniform, considering an origin-destination matrix obtained by assuming that the global level of travel demand is uniformly distributed among the origindestination pairs; 
- estimated, considering the origin-destination travel demand matrix estimated by means of a multi-step model.

The estimated NFDs of the three clusters and of the whole network in the two scenarios are reported in figures 3 and 4. Figure 3 depicts the NFDs for the scenario with uniform travel demand. The diagram of cluster $\mathrm{B}$, associated to the central district, presents the highest values of $\mathrm{AF}\left(\mathrm{AF}_{\max }\right.$ between 250 and $300 \mathrm{vehic} / \mathrm{h})$ and of $\mathrm{AD}\left(\mathrm{AD}_{\max }\right.$ between 30 and $\left.40 \mathrm{vehic} / \mathrm{km}\right)$ than the ones of clusters A, C and of the whole network. The shape of the NFD of the whole network has dimensions within the ones of cluster B and the ones of clusters A and C. This result is in line with the definition of indicators of eqs. (1) and (2), where values increase with increasing values of vehicular flows and densities. In the simulated transport system, the levels of flows and densities of links belonging to cluster B are higher, on average, than the levels of flows and densities of links belonging to clusters $\mathrm{A}$ and $\mathrm{C}$.

Figure 4 presents the NFDs for the scenario with estimated travel demand. The general pattern is similar to one previously descripted related to the scenario with uniform travel demand. The diagram of cluster $\mathrm{B}$ has the highest values of AF $\left(\mathrm{AF}_{\max }\right.$ greater than $\left.300 \mathrm{vehic} / \mathrm{h}\right)$ and of $\mathrm{AD}\left(\mathrm{AD}_{\max }\right.$ around $\left.20 \mathrm{vehic} / \mathrm{km}\right)$ than the ones of clusters A, B and of the whole network. The shape of the NFD for the entire network is, again, within the one of cluster B and the ones of clusters A and $\mathrm{C}$, due to the fact that the levels of flows and densities of links belonging to cluster B are higher, on average, than the levels of links belonging to clusters A and $\mathrm{C}$.

If we compare the two groups of NFDs of Figures 3 and 4, we observe that the maximum values for the whole network in the uniform demand scenario: $\left\{\mathrm{AF}_{\max }\right.$, $\left.\mathrm{AD}_{\max }\right\} \approx\{220,15\}$ are higher than the ones in the estimated demand scenario: $\left\{\mathrm{AF}_{\max }, \mathrm{AD}_{\max }\right\} \approx\{180,10\}$. Moreover, the four NFDs in the uniform demand scenario are 'less dispersed' than the ones in the estimated scenario. The above evidences suggest to us that the shape of the NFD could be influenced by the spatial configuration of the travel demand flows. In the uniform scenario, the spatial distribution of flows and densities should be 'more uniform', and then with less spatial variance, than the case of the estimated scenario. This effect could be mitigated by the fact that in the urban network of Villa San Giovanni the network historically developed according to the levels and spatial structure of the travel demand flows. Therefore, the assignment of a uniform (not-real) origindestination matrix to the real network generates abnormal values of flow/capacity on some links located in peripheral positions of the network.

The diagrams of Figure 5 show the values of maximum link flow vs density experienced in the network for each time slice ( 5 minutes). The four diagrams are calculated in the estimated travel demand scenario and they are related to the set of links belonging to the zones A, B, C and to the whole network. The observation of the four diagrams suggests us the following comments. There are some links reaching a value of maximum flow of 1500 vehic/hour in the diagrams of zone B (the most congested central district) and of the whole network, while the value of 1000 vehic/hour is reached in the diagrams of zone A and C (less congested suburbs). If we compare the values of the maximum link flows of Figure 5 with 


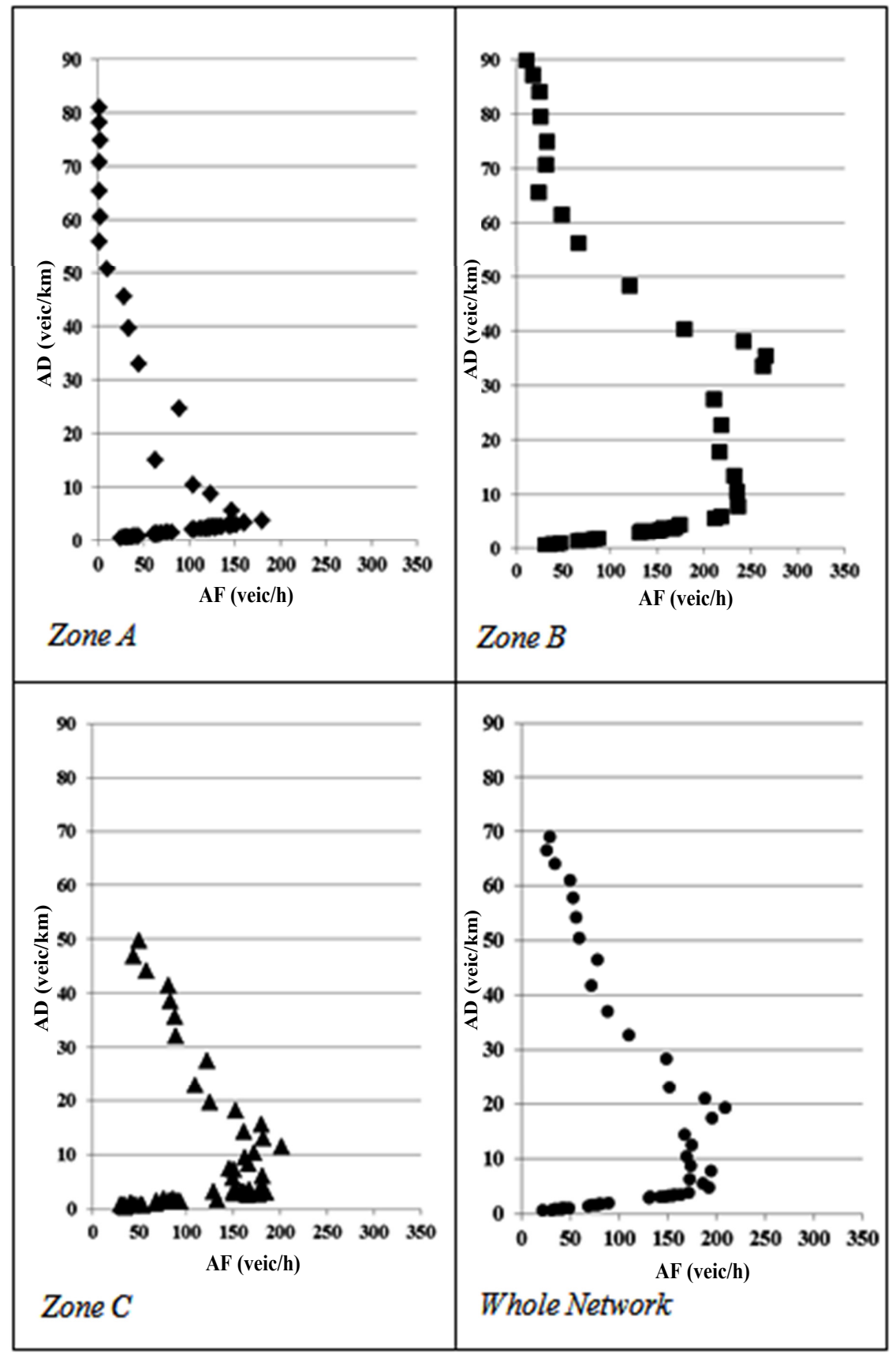

Figure 2: NFDs of zones A, B, C and of the whole network. Uniform travel demand. 


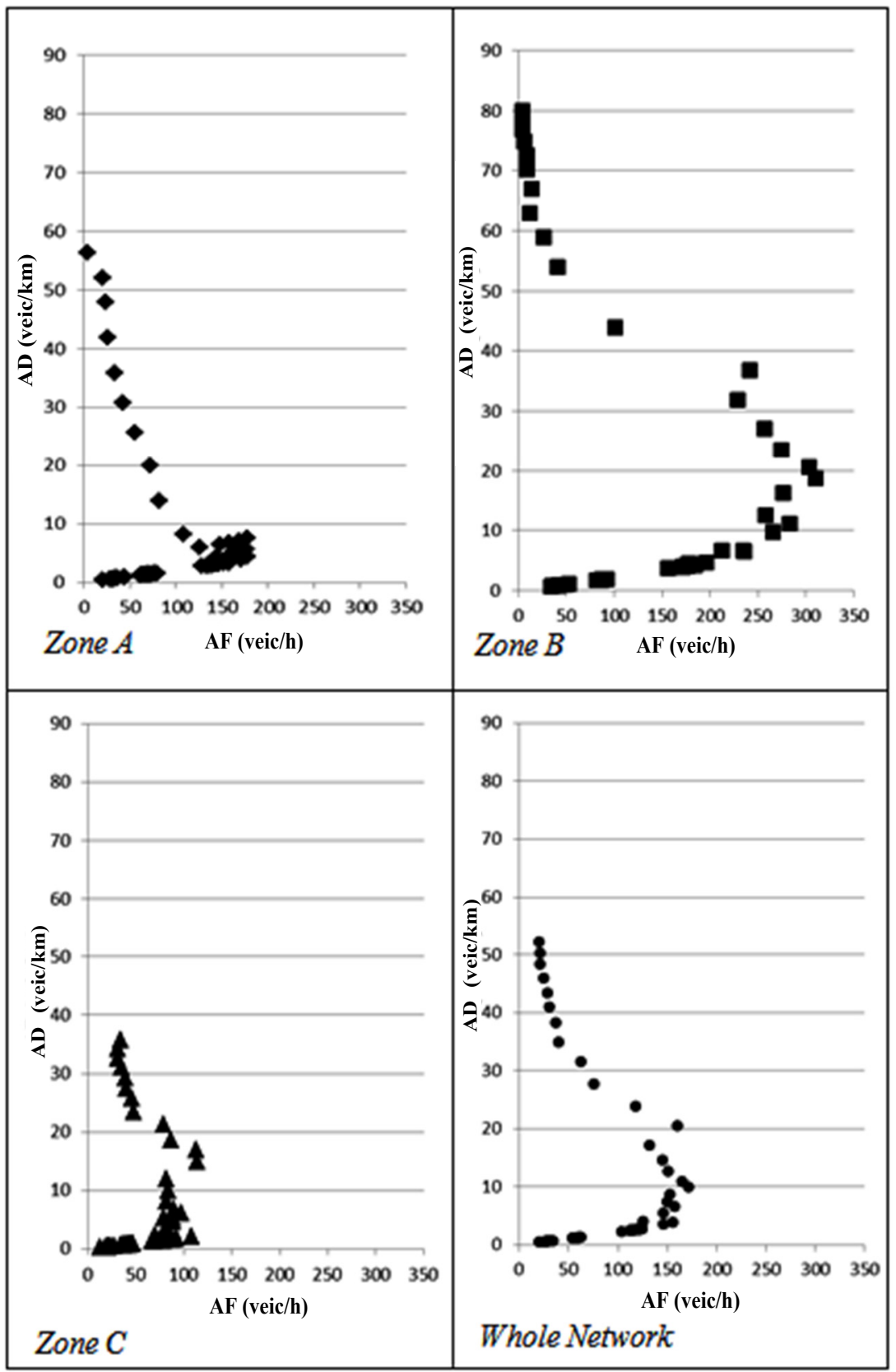

Figure 3: NFDs of zones A, B, C and of the whole network. Estimated travel demand. 
964 The Sustainable City IX, Vol. 2

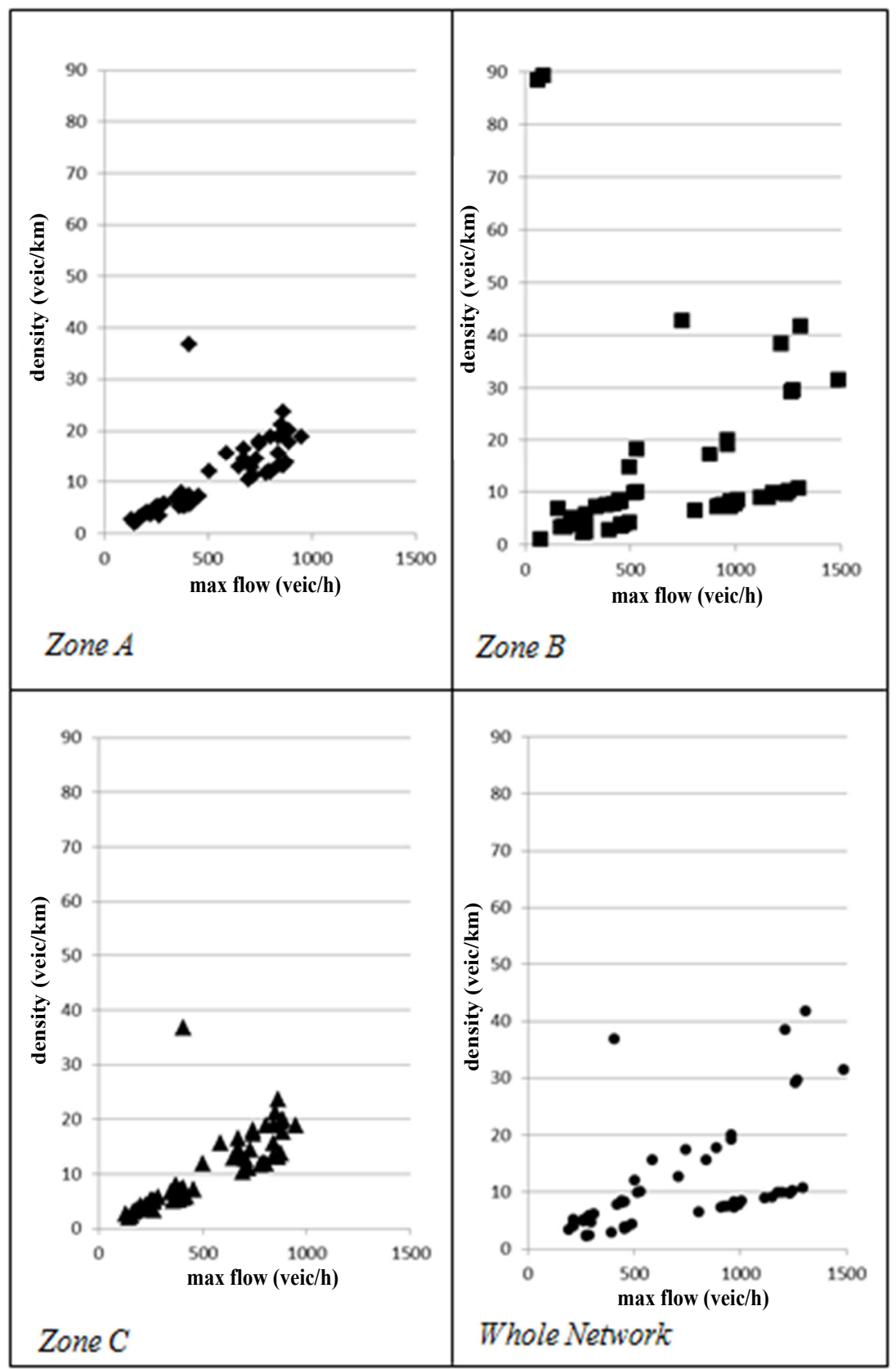

Figure 4: Maximum link flow vs link density for each time slices. Zones A, B, $\mathrm{C}$ and whole network. 
the values of $\mathrm{AF}_{\max }$ in the NFDs of Figure 4, we have a confirmation of the high variance of vehicular flows in the examined urban network compared with the average 'network capacity' (or the capacity of a portion of it).

\section{Conclusions}

The objective of the paper is the estimation of Network Fundamental Diagrams (NFDs) related to portions of network (clusters). The network partitioning is necessary, according to us, in order to reduce the variance of link densities and flows within each cluster, ensuring that the hypothesis of existence of a homogeneously shaped (with no hysteresis) NFD holds.

The experimentation is developed in the town of Villa San Giovanni, in southern Italy. At this stage, the network is partitioned according to the criterion of the spatial distribution of urban activities. The preliminary results suggest us that the shape of the NFD could be influenced by the spatial configuration of the travel demand flows. The method of NFDs estimation on a partitioned network seems also to provide meaningful support in the application of routing strategies (see [10]).

Future research will pursue two main directions. The first one concerns further investigations of the effects on the shape of NFDs generated by different spatial structures (distribution) of travel demand. The second one regards the application of the method of NFDs estimation on a partitioned network on other experimental urban contexts.

\section{References}

[1] Musolino, G. \& Vitetta, A., Short-term forecasting in road evacuation: calibration of a travel time function. WIT Transactions on the Built Environment, 116, pp. 615-626, 2011.

[2] Daganzo, C.F., Urban gridlock: Macroscopic modeling and mitigation approaches. Transportation Research Part B, 41(1), pp. 49-62, 2007.

[3] Geroliminis, N. \& Daganzo, C.F., Existence of urban-scale macroscopic fundamental diagrams: Some experimental findings. Transportation Research Part B, 42(9), pp. 759-770, 2008.

[4] Geroliminis, N. \& Sun, J., Properties of a well-defined macroscopic fundamental diagram for urban traffic. Transportation Research Part B, 45(3), pp. 605-617, 2011.

[5] Buisson, C. \& Ladier, C., Exploring the impact of homogeneity of traffic measurements on the existence of macroscopic fundamental diagrams. Transportation Research Record, 2124, pp. 127-136, 2009.

[6] Ji, Y. \& Geroliminis, N., On the spatial partitioning of urban transportation networks. Transportation Research Part B, 46(10), pp. 1639-1656, 2012.

[7] Keyvan-Ekbatani, M., Kouvelas, A., Papamichail, I. \& Papageorgiou, M., Exploiting the fundamental diagram of urban networks for feedback-based gating. Transportation Research Part B, 46, pp. 1393-1403, 2012. 
[8] Keyvan-Ekbatani, M., Papageorgiou, M. \& Papamichail, I., Urban congestion gating control based on reduced operational network fundamental diagrams. Transportation Research Part C, 33, pp. 74-87, 2013.

[9] Zheng, N., Waraich, R.A., Axhausen, K. W. \& Geroliminis, N., A dynamic cordon pricing scheme combining the Macroscopic Fundamental Diagram and an agent-based traffic model. Transportation Research Part A, 46, pp.1291-1303, 2012.

[10] Musolino, G., Polimeni, A. \& Vitetta, A., Vehicle routing problem in urban networks: an approach based on network fundamental diagram. WIT Transactions on Ecology and the Environment, 179 (ISSN: 1746-448X, Digital ISSN: 1743-3541), 2014.

[11] Cirianni, F., Panuccio, P. \& Rindone, C., A comparison of urban planning systems between the UK and Italy: Commercial development and city logistic plan. WIT Transactions on the Built Environment, 130, pp. 785-797, 2013.

[12] TSS Transport Simulation Systems. AIMSUN User Manual Version 6.1, Barcelona, Spain, 2011.

[13] De Maio, M.L., Musolino, G. \& Vitetta, A., Traffic assignment models in road evacuation. WIT Transactions on Ecology and the Environment, 155, pp. 1041-1051, 2011.

[14] Cascetta, E., Transportation systems engineering: theory and methods. Kluwer Academic Publisher, Dordrecht, The Netherlands, 2001.

[15] Russo, F. \& Vitetta, A., An assignment model with modified Logit, which obviates enumeration and overlapping problems. Transportation, 30(2), pp. 177-201, 2003. 